\title{
Optimized cross-slot microdevices for homogeneous extension
}

\author{
Francisco J. Galindo-Rosales, ${ }^{* a}$ Mónica S. N. Oliveira ${ }^{b}$, and Manuel A. Alves ${ }^{a}$
}

Microfluidic cross-slot devices can generate wide regions of vorticity-free strong extensional flow near the stagnation point, resulting in large extensional deformation and orientation of the microstructure of complex fluids, with possible applications in extensional rheometry and hydrodynamic stretching of single cells or molecules. Standard cross-slot devices, with sharp or rounded corners, generate a flow field with a non-homogeneous extension rate that peaks at the stagnation point, but decays significantly with distance from the stagnation point. To circumvent this limitation, an optimized shape cross-slot extensional rheometer (OSCER) was designed numerically and shown to generate constant extension rate over a wide region of the in- and out-flowing symmetry planes [Haward et al. (2012) Phys. Rev. Lett. 109, 128301]. Since the OSCER device was based on a 2D flow approximation, the practical implementation requires a large aspect ratio, which cannot be reproduced by standard soft-lithography techniques. Here, we propose a set of new designs for optimized cross-slot geometries, considering aspect ratios of order 1 and different lengths of the homogeneous inlet/outlet-flow regions. Micro-particle image velocimetry experiments were carried out in order to validate the flow kinematics, and the velocity profiles were found to be linear along the in- and outflow centrelines in good quantitative agreement with the numerical predictions.

\section{Introduction}

Among the microdevices that generate stagnation-point flows with strong extensional behaviour, the cross-slot device is arguably the configuration which has attracted most attention due to its simple geometry and easy flow control ${ }^{1}$. The standard cross-slot geometry consists of two channels bisecting orthogonally and is a classical geometry for the generation of extensional flow when the opposing inlets and outlets are supplied with equal flow rates. In this configuration, it is possible to generate a free stagnation point at the centre of the cross, i.e. a singular point of zero flow velocity, which combined with a very large residence time and a finite velocity gradient, allows the accumulation of very high Hencky strains and large extensional stresses near the centre ${ }^{2}$. These features have made this flow configuration very appropriate for the analysis of elastic instabilities ${ }^{3-7}$, extensional rheometry of viscoelastic fluids $1,6,8-11$ and for trapping molecules or cells by hydrodynamic means while subjecting them to a strong extensional deformation rate re $^{12-23}$.

Despite its intrinsic potential, the standard cross-slot geometry is only able to generate a uniform extension rate in a short region near the stagnation point ${ }^{6}$. To circumvent this limitation and provide a geometry with homogeneous extensional flow along a wide region near the stagnation point,

\footnotetext{
* Corresponding author

a Address: Centro de Estudos de Fenómenos de Transporte (CEFT), Departamento de Engenharia Química, Faculdade de Engenharia da Universidade do Porto, Rua Dr. Roberto Frias s/n, 4200-465 Porto, Portugal. Fax: +352 22508 1440; Tel: +351 22508 1079; E-mail: galindo@fe.up.pt; curro@galindorosales.com

${ }^{b}$ Address: James Weir Fluids Laboratory, Department of Mechanical and Aerospace Engineering, University of Strathclyde, Glasgow G1 1XJ, United Kingdom.
}

Haward et al. ${ }^{24}$ investigated an optimized shape cross-slot extensional rheometer (OSCER), with a geometrical shape determined using the numerical optimization scheme developed by Alves ${ }^{25}$. The OSCER device requires an aspect ratio $(H / W)$ of order 10 or above, to generate an approximately two-dimensional (2D) flow that provides a nominally constant extension rate over a spatial domain of about 15 times the width $(W)$ of its arms. Precisely, because the OSCER device is based on a $2 \mathrm{D}$ flow approximation, when fabricated in poly(dimethylsiloxane) (PDMS) by soft lithography techniques that limit the aspect ratio of the channel to moderate values, the influence of the end-walls distort the flow field and the OSCER is not able to generate a uniform well-defined extension rate inside the cross-chamber, preventing its use by a wider community that fabricates low aspect ratio microchannels in PDMS ${ }^{26,27}$. We present here a new set of optimized cross-slot microdevices considering aspect ratios $(A R)$ of order 1 , namely $A R=0.5,1$ and 2 . Because the flow is threedimensional in such devices, hereafter we refer to them as optimized 3D cross-slot microchannels. These designs were fabricated in PDMS by replica mould technique, and the resulting hydrodynamics was validated by means of micro-particle image velocimetry $(\mu \mathrm{PIV})$ experiments carried out over a wide range of Reynolds number $\left(R e \in\left[10^{-2}, 10^{2}\right]\right)$ and compared with those obtained for the standard cross-slot and an OSCERshape device, both fabricated in PDMS with $A R=1$. 


\section{Materials and methods}

\subsection{Optimal shape design methodology}

In this work, we used the algorithm for optimal shape design of viscoelastic fluid flow developed by Alves ${ }^{25}$, which in summary consists of a combination of three major components: a fully-automated mesh generator program; a viscoelastic fluid flow solver ${ }^{28,29}$; and the CONDOR ${ }^{30}$ optimizer. The methodology aims to find the shape of a cross-slot microfluidic device capable of achieving optimal performance, which corresponds to a prescribed objective function. We considered the goal of the optimization to search for the microfluidic device shape that generates an ideal planar extensional flow in the mid-plane with $\left[u_{x}, u_{y}\right]=[-\dot{\varepsilon} x, \dot{\varepsilon} y]=\frac{2 U_{c}}{L_{e x t}}[-x, y]$, where $u_{x}$ and $u_{y}$ are the $x$ - and $y$-velocity components, $\dot{\varepsilon}$ is the strain-rate, $U_{c}$ is the centreline fully-developed velocity at the inlet/outlet channels, and $L_{e x t}$ is the length, measured along the centrelines, where a homogeneous extensional flow with constant strain-rate is observed. Starting from an initial estimate of the design variable vector $\mathbf{X}^{\mathbf{0}}$, which parametrizes the shape of the flow geometry, the initial mesh is generated and the CFD simulation is undertaken. From the numerical solution the objective function is evaluated, and this information is sent to the optimizer (Fig.1a).

This process is repeated for a number of design variable parameters, supplied by the optimizer, until the optimal shape is found. Due to the symmetry of the flow geometry, only the first quadrant of the cross-slot geometry is optimized, and the remaining walls are obtained assuming geometrical symmetry. The initial estimate used corresponds to a rounded cross-slot geometry with a characteristic radius $R$. The CFD flow solver is a finite-volume code developed for simulation of laminar flows of viscoelastic fluids described by differential-type constitutive equations. The numerical technique is described in detail in the works of Oliveira et al. ${ }^{28}$ and Oliveira et al. ${ }^{31}$. The governing equations are those expressing conservation of mass of an incompressible Newtonian fluid, $\nabla \cdot \mathbf{u}=0$, and the momentum equation, $\rho \frac{D \mathbf{u}}{D t}=-\nabla p+\mu \nabla^{2} \mathbf{u}$, with $\frac{D \mathbf{u}}{D t}=$ $\frac{\partial \mathbf{u}}{\partial t}+\mathbf{u} \cdot \nabla \mathbf{u}$ representing the material derivative of $\mathbf{u}, \rho$ is the fluid density, $t$ is the time, $p$ is the pressure and $\mu$ is the dynamic viscosity of the fluid. Creeping flow (Stokes flow) conditions are obtained by neglecting the material derivative term in the left hand side of the previous equation.

In this work, we have optimized seven different cross-slot microchannels, based on three geometric parameters (Fig.1): the radius $(R)$ of the rounded cross-slot geometry used as initial guess, the length where an uniform extension rate $\left(L_{e x t}\right)$ is required along the centrelines and the aspect ratio of the microchannel $(A R=H / W)$. All the geometries are defined in Table 1 , where $H$ represents the channel depth and $W$ the

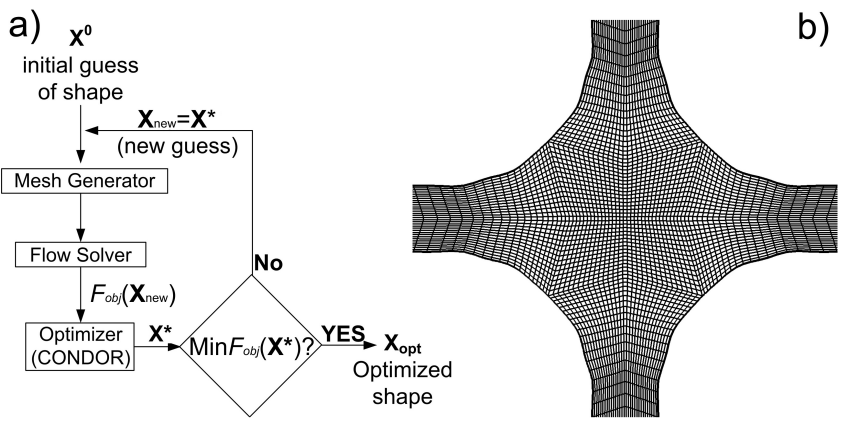

c)

d)


Fig. 1 Optimal shape design procedure. a) Schematic illustration of the optimization flowchart, b) top-view of an exemplifying optimized design, c) 3D illustration of the optimized design including the geometric parameters, d) target velocity and strain-rate profiles along the vertical centreline $(x=0)$.

inlet/outlet channel widths*. Thus, for instance, the geometry named as L2R2.5AR1 has been optimized for a value of $L_{e x t}=2 W$, a radius $R=2.5(W / 2)$ and for an aspect ratio of $H / W=1$.

Fig. 2 shows the normalized velocity profile $\left(u_{y} / U\right)$ along the vertical (outflow) centreline at different $z$-planes, where $U$ is the bulk velocity in the inlet/outlet channels, and the corresponding extension rate $(\dot{\varepsilon})$ profile resulting from the $3 \mathrm{D}$ optimization of cross-slot microchannel using a Newtonian fluid under creeping flow conditions. These profiles follow the ex-

* As an example, the profiles of the geometries L5R8AR0.5, L5R8AR1 and L5R8AR2 are provided as Electronic Supplementary Information.

Table 1 Characteristic dimensions of the cross-slot micro devices.

\begin{tabular}{lllll}
\hline 3D optimized cross-slot & $H[\mu \mathrm{m}]$ & $W[\mu \mathrm{m}]$ & $L_{e x t}[\mu \mathrm{m}]$ & $R[\mu \mathrm{m}]$ \\
\hline a)L2R2.5AR1 & 100 & 100 & 200 & 125 \\
b)L3R4AR1 & 100 & 100 & 300 & 200 \\
c)L5R6AR1 & 100 & 100 & 500 & 300 \\
d)L5R8AR0.5 & 50 & 100 & 500 & 400 \\
e)L5R8AR1 & 100 & 100 & 500 & 400 \\
f)L5R8AR2 & 200 & 100 & 500 & 400 \\
g)L10R10AR1 & 100 & 100 & 1000 & 500 \\
h)Standard & 100 & 100 & $\sim 150$ & - \\
i)OSCER-shape & 100 & 100 & 1500 & 1000 \\
\hline
\end{tabular}




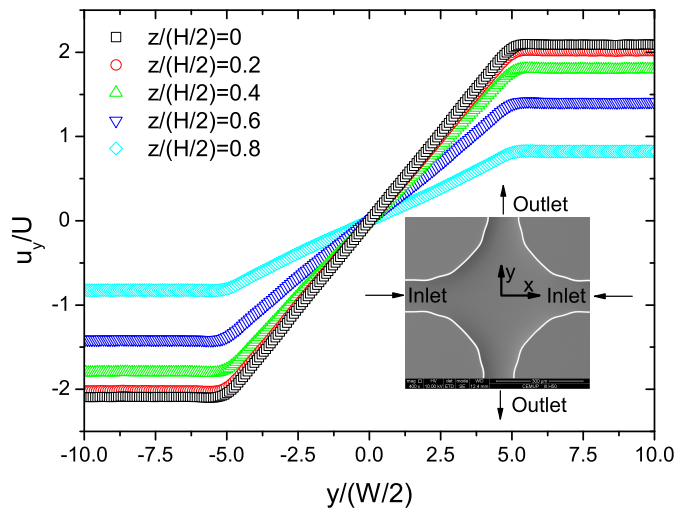

(a)

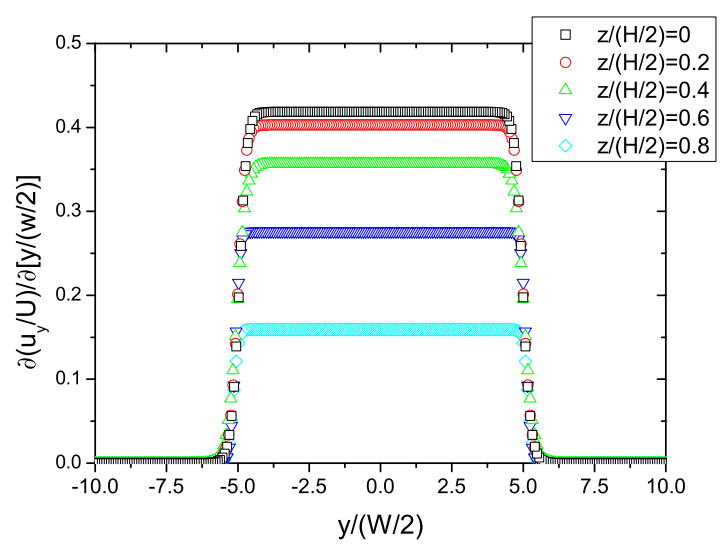

(b)

Fig. 2 Numerical profiles in the 3D optimized cross-slot microchannel $(L 5 R 8 A R 1)$ along the outlet centrelines $(x=0)$ for a range of $z$-planes and for creeping flow. a) Normalized velocity profiles; b) corresponding normalized strain-rate profiles.

pected target functions closely, with a linear variation of the velocity profile in the region of envisaged constant strain-rate and showing sharp edges at the end of this defined zone of constant extension rate, thus providing an excellent control of the extension rate to which the fluid or the object being analysed is subjected to in the central cross chamber of the device. However, there is a clear gradient of extension rate in the $z$ direction, with the strain-rate reaching its maximum value at the mid-plane and decreasing towards the bottom/top walls as a direct consequence of the no-slip at the end-walls. Despite this strain-rate gradient, we should highlight that the uniformity of the extension rate profiles at each z-plane is maintained. The non-negligible gradient of extension rate along the depth of the channel may limit somehow the applicabil-
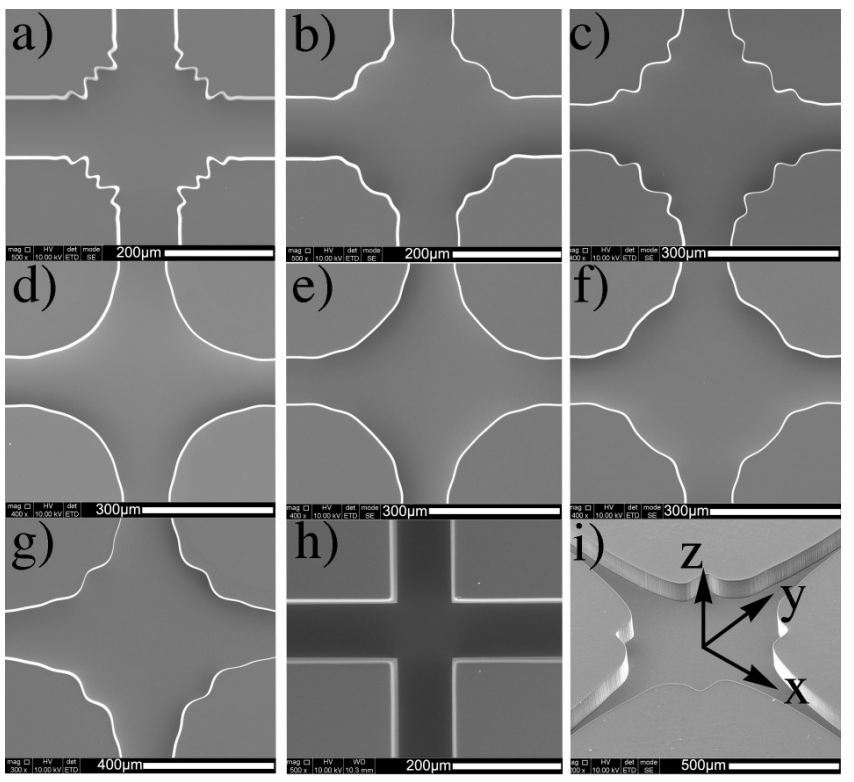

Fig. 3 Scanning electron microscopy (SEM) images of the cross-slots used in this work as described in Table1. In cases a)-h) a top-view is shown, while in case i) a perspective view is illustrated.

ity of these 3D optimized cross-slot for extensional microfluidic rheometry, since it can have repercussions on the extra pressure drop, as well as on flow induced birefringence measurements, which rely on a cumulative measurement of the retardation throughout the light path (depth of the channel). However, we note that near the centre plane the variation of the strain-rate with the depth is moderate, as illustrated in Fig.2(b). For the profiles at $z /(H / 2)=0.2$ and $z /(H / 2)=0.4$, a variation of $20 \%$ and $40 \%$ along $z$ corresponds to a variation of $3.66 \%$ and $14.74 \%$, respectively, in the strain-rate with regards to the centreplane $(z /(H / 2)=0)$. In the particular case of applications involving the stretching of single macromolecules or cells, because these specimens can be trapped in the central region of the cross chamber at the mid-plane, the uniformity of the extension profiles around the mid-plane generated by these 3D optimized cross-slots reveals as a promising leap forward towards the mechanical characterisation of molecules and cells in lab-on-a-chip devices.

\subsection{Experiments}

The optimized shapes were fabricated in PDMS from SU-8 photoresist moulds using standard soft-lithography techniques. A high-resolution chrome mask was employed to obtain high quality SU-8 moulds with nearly vertical sidewalls and well-defined corner features. Fig. 3 shows scanning electron microscopy (SEM) images of all optimized cross-slot microdevices used in the present study. The Cartesian 
coordinate system used in our measurements is located on the mid-plane at the centre of the cross chamber. Using the Matlab $^{\circledR}$ Image Processing Toolbox ${ }^{\mathrm{TM}}$ and bright field microcopy images, we have confirmed that the numerical designs were reproduced in the PDMS channels with a deviation in the dimensions below $5 \%$ of the channel width. The depth of the devices and the channel width of the inlet/outlet arms are indicated in Table1.

The experimental results presented here were obtained using distilled water as working fluid. All the experiments were carried out at an average temperature of $295 \mathrm{~K}$, for which the dynamic viscosity and the density of distilled water are $\mu=0.96 \mathrm{mPa} \cdot \mathrm{s}$ and $\rho=997.8 \mathrm{~kg} / \mathrm{m}^{3}$, respectively.

The flow in the cross chamber was characterized experimentally using $\mu \mathrm{PIV}^{33,34}$ following the procedure detailed by Oliveira et al. ${ }^{35}$. A CCD camera (FlowSense 2M camera) connected to an inverted microscope (Leica DMI 5000 $\mathrm{M}$ ) with $10 \mathrm{X}$ and $20 \mathrm{X}$ objective lenses (numerical aperture, $\mathrm{NA}=0.30$ and $\mathrm{NA}=0.5$, respectively) and a filter cube were used to acquire the images in the mid-plane $(z=0)$. For volume illumination of the flow, we used a double-pulsed Nd:YAG laser system operating at a wavelength of $\lambda=532$ $\mathrm{nm}$. As tracer particles, we used $1 \mu \mathrm{m}$ diameter fluorescent particles (FluoSphere carboxylate-modified, nile red, $\mathrm{Ex} / \mathrm{Em}=535 / 575 \mathrm{~nm}$ ) at a concentration of $\sim 0.02 \%$ by volume. The time lapse between two consecutive frames was adjusted depending on the flow rate. For all cases, a minimum of 100 image pairs were recorded, divided into interrogation areas of $32 \times 32$ pixels. These images were processed and ensemble averaged using the DynamicStudio software (version 2.30.47, Dantec Dynamics).

The flow rate was controlled by means of a neMESYS low pressure syringe pump (Cetoni $\mathrm{GmbH})$ with three independent modules. The flow rates considered ranged from $2 \mu \mathrm{l} / \mathrm{h}$ to $36 \mathrm{ml} / \mathrm{h}$ in order to cover Reynolds numbers from $10^{-2}$ up to $10^{2}$ in all the geometries. Depending on the flow rate different Hamilton syringes $(25 \mu \mathrm{l}, 500 \mu \mathrm{l}$ and $1 \mathrm{ml})$ were used to ensure pulsation-free dosing. Three pumps controlled two inlet and one outlet flow rates, while the remaining outlet was left open to the atmosphere to balance the flow.

\section{Results and Discussion}

The exact length of the extension region $\left(L_{\text {ext }}\right)$ associated with the standard cross-slot is not obvious a priori, but our $\mu \mathrm{PIV}$ measurements revealed that $L_{\text {ext }} \approx 1.5 \mathrm{~W}$ (Fig.4(a)). For that reason, we compare directly the standard cross-slot with the geometry optimized using a similar $L_{\text {ext }}(L 2 R 2.5 A R 1)$ to compare their performances. Fig.4(b) shows the velocity profiles obtained for the $3 \mathrm{D}$ optimized cross-slot $L 2 R 2.5 A R 1$ at $R e$ ranging from 0.025 to 5 , revealing that the length of quasiuniform strain-rate is indeed $L_{e x t} \approx 2 W$. Fig.4(c) compares the velocity profiles between both cross-slots at $R e=0.75$ and $R e=5$. It is evident that the profile in the 3D optimized crossslot is clearly linear (within experimental uncertainty) in the zone of extension, following the numerically predicted profiles, while the one given by the standard cross-slot shows a sigmoidal shape. By means of numerical derivatives we calculated the extension rate profiles along the outlet centreline ( $\dot{\varepsilon}=\frac{\partial u_{y}}{\partial y}$ ), and Fig.4(d) clearly shows that the extension rate profile is significantly more uniform for the optimized crossslot. Given the typical small oscillations observed in the experimental results, due to the experimental errors of the $\mu \mathrm{PIV}$ technique, the calculation of the strain-rate from the derivative of the velocity profile requires a careful analysis. To avoid the high (spatial) frequency oscillation in the computed strain-rate that would occur, we fitted the velocity profiles according to the general expression $u_{y}=\frac{1}{\left[(1 / a y)^{p}+(1 / b)^{p}\right]^{1 / p}}$ and determined the parameters $a, b$ and $p$ that minimize the square of the differences between the fitted expression and the experimental measurements. This function has the asymptotes $u_{y}=a y$ for small $|y|$ and $u_{y}=b$ for $|y| \gg L_{e x t}$, as expected for the optimized shape. The strain-rates shown in Fig.4(d) are computed from the derivative of this fitted function.

As the optimization was carried out for creeping flow conditions, the uniformity of the strain-rate profiles and the length of uniform extension is only close to expected values at low $R e$. When the flow conditions deviate significantly from inertialess flow conditions the strain-rate field becomes less homogeneous ( $R e \gtrsim 10)$, even for the channels with the largest aspect ratio as shown in Fig.5. This does not mean that it is impossible to achieve a uniform extension rate at higher values of $R e$, but instead it requires to optimize a new geometry taking into account the convective terms in the momentum equation. However, it is not practical to have different geometries for different values of $R e$ and therefore we have restricted our analysis to low $R e$, since these conditions are easily met and are relevant for microfluidics.

Fig.6(a) shows a comparison between the normalized velocity profiles measured for all the $3 \mathrm{D}$ optimized cross-slot microdevices, the OSCER-shaped device and the standard crossslot. At $A R=1$, the OSCER-shaped device and the crossslot are clearly not as effective in obtaining a homogeneous flow field along the outlet centrelines, while the velocity profiles in the extension dominated region are clearly linear for all the optimized devices and the region of uniform extension rate for each one has the predicted length. As a consequence, playing with the flow rate $(Q)$ and the length of the extension region $\left(L_{\text {ext }}\right)$, it is possible to reach whichever position in the $\dot{\varepsilon}-R e$ parameter space (Fig.6(b)) required, as long as $R e$ is low $(R e \lesssim 10)$. Thus, with our optimal shape design approach 


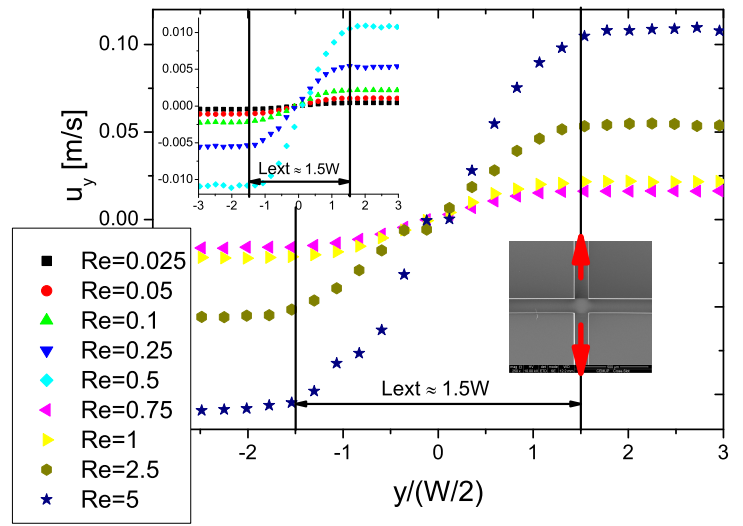

(a)

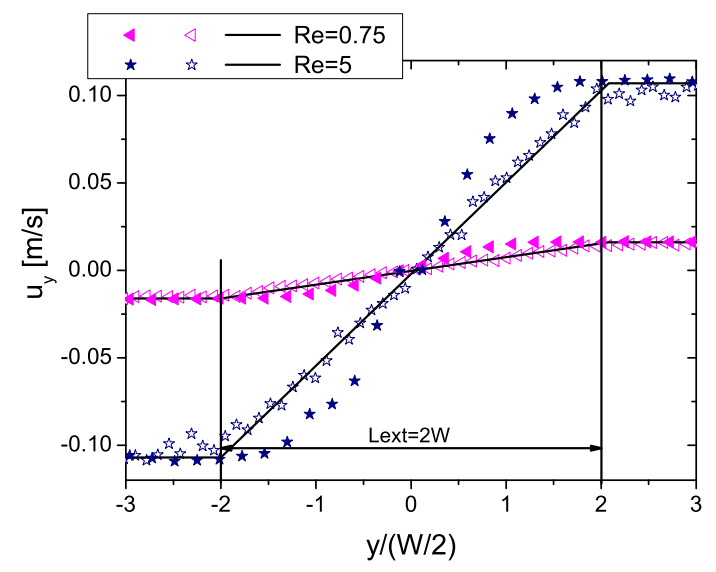

(c)

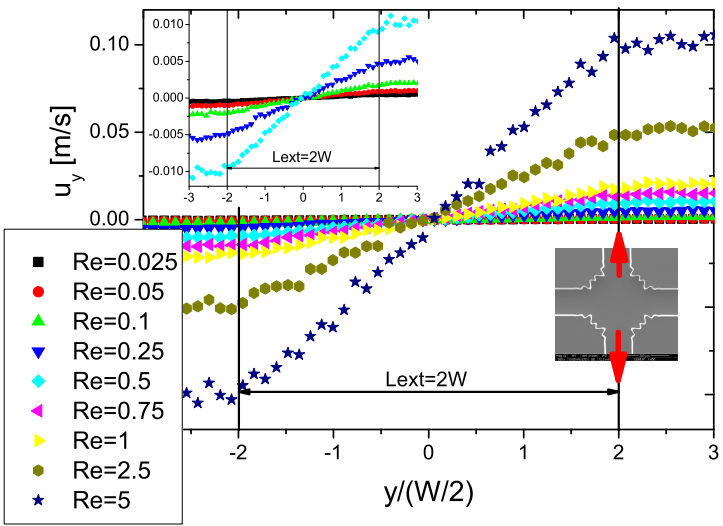

(b)

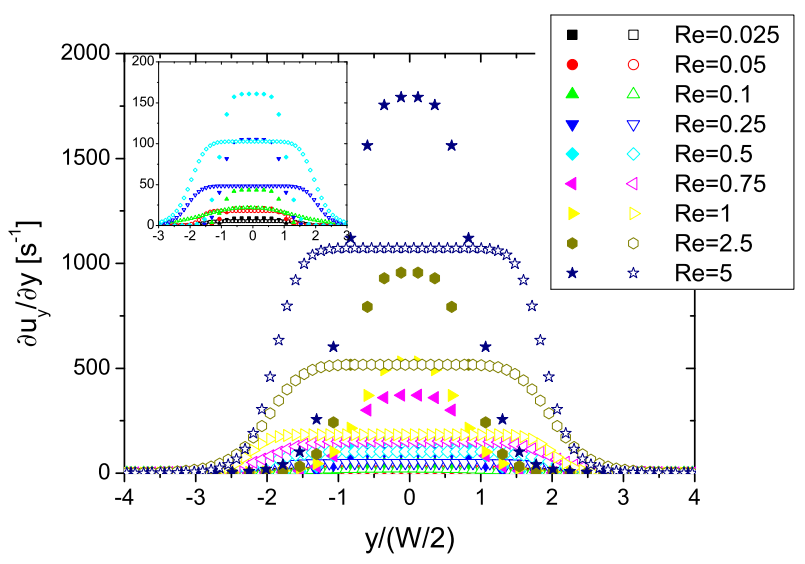

(d)

Fig. 4 Standard cross-slot $\boldsymbol{v s}$. 3D optimized cross-slot ( $L 2 R 2.5 A R 1)$ : Velocity profiles measured at the mid-plane along the centreline $(x=0, z=0)$ for Reynolds numbers ranging from 0.025 to 5 in (a) the standard cross-slot, and (b) the 3D optimized cross-slot; (c) comparison between the target velocity profiles (lines) and those obtained experimentally for the standard cross-slot (solid symbols) and the 3D optimized cross-slot (hollow symbols) at $R e=0.75$ and 5; (d) comparison between the extension rate $(\dot{\varepsilon})$ generated for the standard cross-slot (solid symbols) and the 3D optimized cross-slot (hollow symbols) for Reynolds numbers ranging from 0.025 to 5.

it is possible to create a 3D optimized cross-slot device on demand depending on the requirements for controlled extensional flow.

\section{Conclusions}

A range of 3D optimized cross-slot devices were designed numerically in order to generate homogeneous extensional flows. The devices were optimized considering aspect ratios of order 1, with the purpose of exploring the widespread use and the benefits of soft lithography techniques for microfabrication. These 3D optimized cross-slots showed a significantly improved performance in comparison with the standard cross- slot, in which the flow field exhibits a nonhomogeneous extension rate that peaks at the stagnation point. The enhanced homogeneity of the extensional flow in the optimized designs, where the velocity varies linearly with distance from the centre in a wide region along the inflow/outflow centrelines, has great potential for a number of applications, such as those related to extensional micro-rheometry or mechanical characterisation of droplets, molecules or cells by means of hydrodynamic stretching. Depending on the application and the range of strain-rates required, the most appropriate geometry can be selected or tuned on demand, highlighting the potential of our shape-optimization approach for efficient microfluidic device design. 




Fig. 5 Working away from the optimized flow conditions. Normalized velocity profiles measured along the inlet (hollow symbols) and outlet (semi-solid symbols) centrelines at the mid-plane $(z=0)$ for non-creeping flow conditions in the 3D optimized cross-slot $L 5 R 8 A R 2$.

\section{Acknowledgements}

MMA acknowledges FCT, FEDER and COMPETE through project PTDC/EME-MFE/114322/2009. FJG-R was supported by Fundação para a Ciência $e$ a Tecnologia (FCT), COMPETE and FEDER through scholarship SFRH/BPD/69663/2010. MSNO acknowledges EPSRC funding through grant EP/L013118/1. The authors would also like to acknowledge Dr. Simon J. Haward and Dr. L. CampoDeaño for helpful discussions and comments.

\section{References}

1 F. J. Galindo-Rosales, M. A. Alves and M. S. N. Oliveira, Microfluidics Nanofluidics, 2013, 14(1-2), 1-19.

2 S. J. Haward and G. H. McKinley, Physical Review E, 2012, 85, 031502.

3 P. E. Arratia, C. C. Thomas, J. Diorio and J. P. Gollub, Physical Review Letters, 2006, 96, 144502.

4 A. M. Afonso, M. A. Alves and F. T. Pinho, Journal of Non-Newtonian Fluid Mechanics, 2010, 165, 743-751.

5 S. J. Haward, Rheologica Acta, 2010, 49, 1219-1225.

6 S. J. Haward, T. J. Ober, M. S. N. Oliveira, M. A. Alves and G. H. McKinley, Soft Matter, 2012, 8, 536-555.

7 N. Dubash, P. Cheung and A. Shen, Soft Matter, 2012, 8, 5847-5856.

8 J. A. Odell and S. A. Carrington, Journal of non-Newtonian Fluid Mechanics, 2006, 137, 110-120.

9 S. J. Haward, J. A. Odell, M. Berry and T. Hall, Rheologica Acta, 2011, 50, 869-879.

10 S. J. Haward, V. Sharma and J. A. Odell, Soft Matter, 2011, 7, 9908-9921.

11 G. Juarez and P. E. Arratia, Soft Matter, 2011, 7, 9444-9452.

12 E. S. G. Shaqfeh, Journal of Non-Newtonian Fluid Mechanics, 2005, 130, $1-28$.

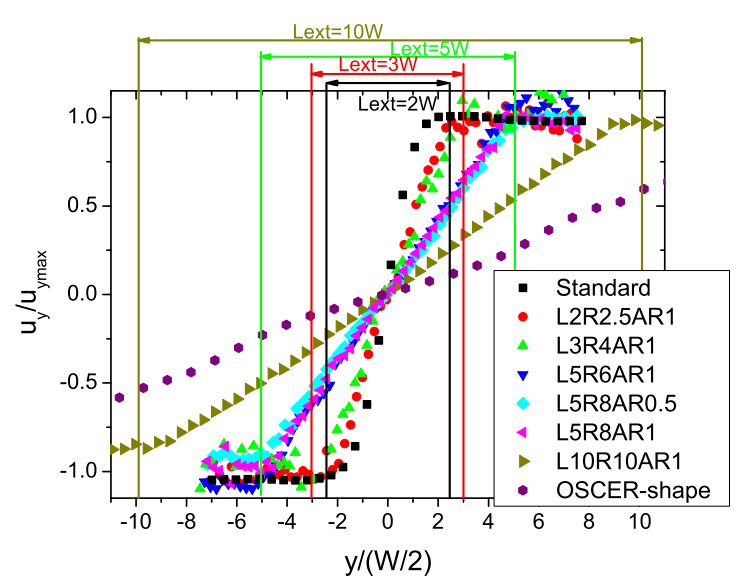

(a)

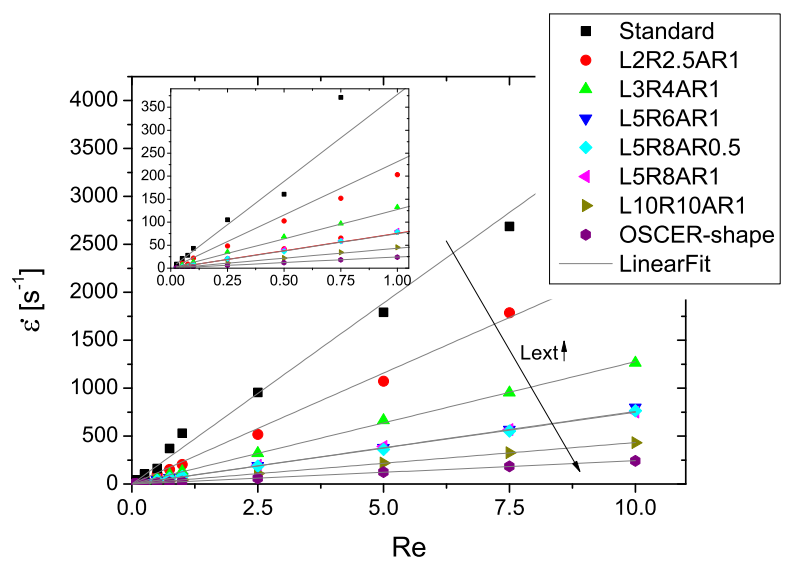

(b)

Fig. 6 Length of the extension regions in the 3D optimized cross-slots.a) Normalized velocity profiles measured at the mid-plane $(z=0)$ for each 3D optimized cross-slot at $R e=0.1$. b) Extension rate $(\dot{\varepsilon})$ generated for the $3 \mathrm{D}$ optimized cross-slots, the standard cross-slot and the OSCER-shape as a function of $R e$.

13 A. G. Balducci, J. Tang and P. S. Doyle, Macromolecules, 2008, 41, 9914 9918.

14 R. Dylla-Spears, J. E. Townsend, L. Jen-Jacobson, L. L. Sohn and S. J. Muller, Lab on a Chip, 2010, 10, 1543-1549.

15 M. Tanyeri, E. M. Johnson-Chavarria and C. M. Schroeder, Applied Physics Letter, 2010, 96, 224101.

16 M. Tanyeri, M. Ranka, N. Sittipolkul and C. M. Schroeder, Lab on a Chip, 2011, 11, 1786-1794.

17 M. Curtis, G. J. Sheard and A. Fouras, Lab on a Chip, 2011, 11, $2343-$ 2351.

18 D. Gossett, T. H.T.K., S. Lee, Y. Ying, A. Lindgren, O. Yang, J. Rao, A. Clark and D. Di Carlo, Proceedings of the National Academic of Science, 2012, 109(20), 7630-7635.

19 W. Xu and S. J. Muller, Lab on a Chip, 2012, 12, 647-651.

20 W. Xu and S. J. Muller, Lab on a Chip, 2012, 11, 435-442.

21 D. J. Mai, C. Brockman and C. M. Schroeder, Soft Matter, 2012, 8, 
10560-10572.

22 J. Dudani, D. Gosset, H. Tse and D. Di Carlo, Lab on a Chip, 2013, 13, 3728-3734.

23 P. A. May and J. S. Moore, Chem. Soc. Rev., 2013, 42, 7497-7506.

24 S. J. Haward, M. S. N. Oliveira, M. A. Alves and G. H. McKinley, Physical Review Letters, 2012, 109, 128301.

25 M. A. Alves, AIP Conference Proceedings, 2008, 1027, 240-242.

26 D. C. Duffy, J. C. McDonald, J. A. Schueller and G. M. Whitesides, Analytical Chemistry, 1998, 70, 4974-4984.

27 J. C. McDonald, D. C. Dufy, J. R. Anderson, D. T. Chiu, H. Wu and G. M. Whitesides, Electrophoresis, 2000, 21, 27-40.

28 P. J. Oliveira, F. T. Pinho and G. A. Pinto, Journal of Non-Newtonian Fluid Mechanics, 1998, 79(1), 1-43.

29 M. A. Alves, P. J. Oliveira and F. T. Pinho, International Journal for Numerical Methods in Fluids, 2003, 1027, 47-75.

30 F. V. Berghen and H. Bersini, Journal of Computational and Applied Mathematics, 2005, 181, 157-175.

31 M. S. N. Oliveira, F. T. Pinho and M. A. Alves, Journal of Fluid Mmechanics Nanofluidics, 2012, 711, 171-191.

32 R. B. Bird, R. C. Armstrong and O. Hassager, Dynamics of polymer liquids. Volume 1 - Fluid mechanics. Second edition., John Wiley and Sons Inc., United States, 1987.

33 J. G. Santiago, S. T. Wereley, C. Meinhart, D. J. Beebe and R. J. Adrian, Experiments in Fluids, 1998, 25, 316-319.

34 C. D. Meinhart, S. T. Wereley and M. H. B. Gray, Measurement Science and Technology, 2000, 11, 809-814.

35 M. S. N. Oliveira, M. A. Alves, F. T. Pinho and G. H. McKinley, Experiments in fluids, 2007, 43, 437-451. 